\title{
Desarrollo de sitios web para la oferta de servicios característicos de la Administración electrónica
}

\author{
Por Pablo Lara Navarra y José Ángel Martínez Usero
}

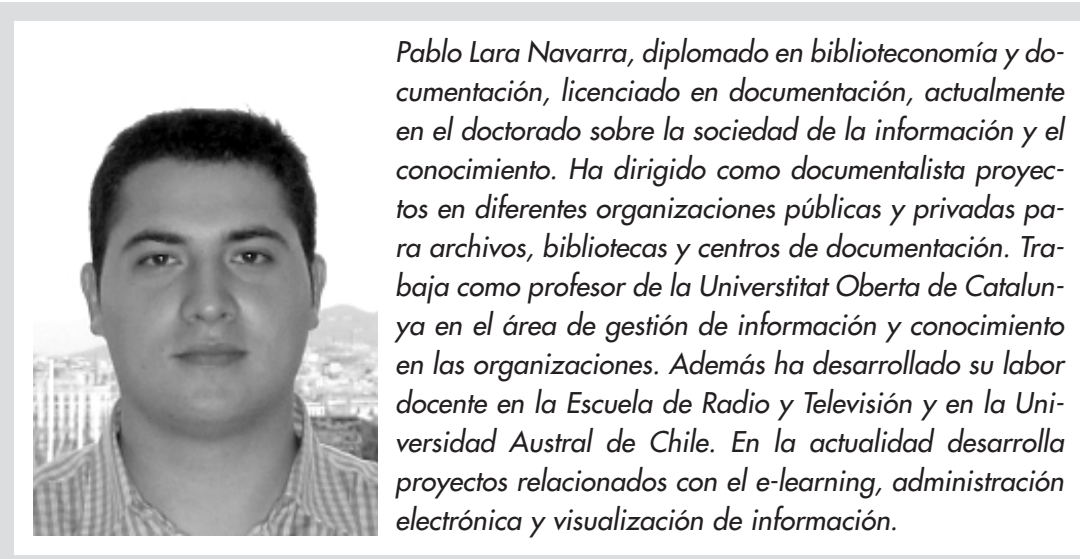

Resumen: Se establece una demarcación del concepto de administración electrónica y su evolución, se mencionan las diferentes perspectivas y aplicaciones de la administración electrónica. Se identifican los pilares básicos para su desarrollo en el ámbito europeo: desarrollo tecnológico, legislativo y metodológico. Se exponen ciertos aspectos relacionados con la metodología para el desarrollo de sitios web públicos, ofreciendo una serie de directrices y buenas prácticas.

Palabras clave: Administración electróni-

José Ángel Martínez Usero, diplomado en biblioteconomía y documentación, licenciado en documentación, master en sistemas de información y doctorado en gestión de las Ti en las organizaciones. Ha trabajado como documentalista en diferentes organizaciones privadas $y$ como website manager para Calderdale $\mathrm{MBC}$ (UK). Trabaja en el desarrollo de Portal Mayores (Csic), como consultor de la licenciatura en documentación de la UOC y profesor asociado de la Universidad Carlos III. Actualmente desarrolla proyectos relacionados con la gestión del conocimiento y la administración electrónica.

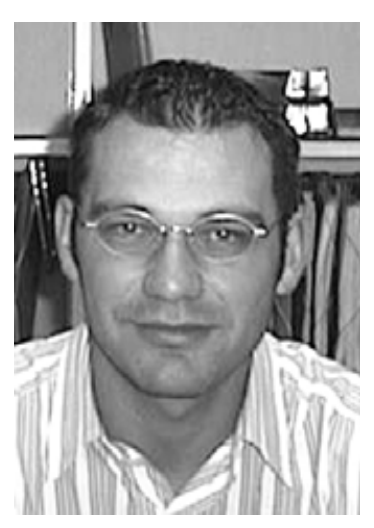
ca, Gobierno digital, Información pública, Sitios web, Gestión web, Tecnologías de la información y la comunicación, TIC.

Title: Web site development techniques for providing e-government services

Abstract: The conceptual framework for e-government and its historical evolution are explained, including mention of different approaches and applications. The essential pillars for developing e-government services at the European level are identi-

fied as technological, legal, and methodological. Finally, issues are discussed regarding the recommended methodology for developing government web sites, with suggested guidelines and good practices.

Keywords: E-government, Digital government, Public information, Web sites, Web management, Information and communication technologies, ICT.

Lara Navarra, Pablo; Martínez Usero, José Ángel. "Desarrollo de sitios web para la oferta de servicios característicos de la administración electrónica". En: El profesional de la información, 2003, mayo-junio, v. 12, n. 3, pp. $190-199$.

\section{El concepto de administración electrónica (e-government)}

Este término fue utilizado por primera vez en un informe del Government Information Technology Services Board del gobierno de los EUA en febrero de

Los autores quieren agradecer la colaboración de los directores y el equipo de desarrollo de Portal Mayores en la elaboración de este artículo.

http://www.imsersomayores.csic.es
1997 (Relyea, 2002, p. 9). En su uso inicial era poco más que el reconocimiento de la influencia de las tecnologías de la información, su uso y aplicación en las organizaciones gubernamentales. Posteriormente empezó a ser aplicado también a la meta política de llevar a cabo servicios gubernamentales más eficientes y con menor coste.

Inicialmente se definió e-government, gobierno electrónico, gobierno digital, e-gobierno, o bien, el término más aceptado y difundido, administración electrónica (elprincipe.com), como la puesta a disposición de los ciudadanos a través de internet de información 
sobre acciones de gobierno, es decir información estática en la Red sobre la gestión gubernamental. Con posterioridad se fueron incorporando algunos trámites en línea con el fin de agilizar la atención y disminuir las esperas y las largas filas en los centros de atención estatales. Así, todavía en muchos lugares se entiende como administración electrónica la mejora de la atención al ciudadano mediante el uso de las tecnologías de información y comunicación (TIC). Pero ésta es una visión parcializada del concepto. La noción actual es una idea mucho más integral que concibe el uso de las tecnologías de información y comunicación con el fin de construir una nueva forma de organización social en redes, interconectada y horizontal (e-governance).

Devadoss realiza una revisión del concepto y propone una definición genérica en la que se determinan dos formas de transformación del gobierno tradicional: por un lado un cambio en la gestión pública mejorando, por ejemplo, la calidad del servicio, reduciendo los costes y renovando los procesos administrativos. Por otra parte, la conversión del gobierno mediante el análisis del funcionamiento de los procesos democráticos.

A partir de un concepto tan amplio, a la hora de entender las iniciativas de administración electrónica podemos encontrarnos con las siguientes perspectivas (Lenk; Traunmuller, 2000):

-De gestión: considera el uso de las tecnologías de la información para mejorar el funcionamiento de la administración.

-Del ciudadano: describe la posición del usuario final que recibe los servicios de administración electrónica.

-De conocimiento: concibe a los trabajadores de la administración como una fuente de conocimiento corporativo que se puede gestionar.

-Del proceso: analiza la utilización de las tecnologías de la información para mejorar los servicios electrónicos.

-De la tele-cooperación: trata sobre la interacción de los diferentes agentes implicados en los proyectos de administración electrónica.

En el presente artículo nos centraremos casi exclusivamente en la denominada perspectiva de proceso, puesto que se proporcionará un conjunto de directrices y buenas prácticas en el desarrollo de sitios web para la oferta de servicios característicos de la administración electrónica.

Las aplicaciones de las tecnologías de la información para la mejora de los servicios públicos (perspectiva de proceso), se pueden dividir en 3 categorías (Marchionini, 2003, pp. 25-27): acceso a la informa- ción, servicios transaccionales y participación ciudadana. Esta distribución encaja perfectamente con la visión europea en materia de administración electrónica, que realiza la siguiente división (Fundación Retevisión, 2002a, p. 6):

—Información del sector público (contenidos): legislación, transporte, turismo, etc.

- Servicios públicos electrónicos (comercio electrónico): recaudatorios, registrales, permisos y licencias, facilidades, etc.

-Democracia electrónica (participación ciudadana): encuestas, procesos de consulta pública, voto electrónico, etc.

\section{Las bases de la administración electrónica}

La administración electrónica en la agenda de la UE tiene un gran peso pero está lejos de presentar un enfoque coordinado, probablemente porque la iniciativa en el implementación de servicios se ha dejado como una responsabilidad individual de cada estado miembro y, por tanto, cada país ha evolucionado de una forma diferente. Según el estudio de Strejcek y Theil (Strejcek; Theil, 2002, pp. 309-313) se pueden diferenciar dos tendencias claras: por un lado, el conjunto de países que apuesta por un desarrollo tecnológico y, por otro, aquellos que hacen mayor hincapié en una legislación aplicable a los diferentes procesos, tecnologías y metodologías que tienen lugar en el desarrollo de servicios característicos de la administración electrónica. Podríamos añadir un tercer grupo diferenciador: los que han desplegado un conjunto de directrices, normas y buenas prácticas para llevar a cabo un desarrollo armónico de los servicios (desde el punto de vista tecnológico y metodológico) y las plataformas tecnológicas que los hacen posibles.

Podríamos decir que la administración electrónica se asienta sobre 3 bases fundamentales: el marco tecnológico, el legislativo y el metodológico. La mayoría de los estados miembros están en proceso de ajustar los dos primeros pilares, pero existe un abandono casi generalizado del tercero, a pesar de los esfuerzos de la UE en la generación de informes de evaluación comparativa (benchmarking) y una política orientada a difundir buenas prácticas europeas.

Una de las conclusiones esenciales de las Jornadas sobre administración electrónica de 2001 con respecto al desarrollo de sitios web consiste en la necesidad de "establecer políticas comunes para homogeneizar en la medida de lo posible los objetivos y contenidos de los portales de las administraciones públicas. Manuales de estilo corporativos, normalización de url (uniform resource locator), acceso a discapacitados, 
intercambio de documentos entre administraciones, etc., para dar mayores facilidades a los ciudadanos a la hora de utilizar servicios electrónicos".

Para la creación de los contenidos que a continuación se presentan se han tenido en cuenta las directrices contenidas en los siguientes documentos:

- Guidelines for UK government websites. http://www.e-envoy.gov.uk/oee/oee.nsf/sections/ guidelines-top/\$file/guidelines_index.htm

—Directrices del Consejo Superior de Informática. Sección documentos. http://www.map.es/csi/pg6000.htm

\section{Los 5 retos de la administración electrónica}

\section{Canales y medios de acceso múltiples}

En el futuro cercano, el ordenador personal no será la única vía de interacción electrónica con las organizaciones públicas. Los usuarios podrán elegir la tecnología con la que quieren acceder a los servicios disponibles, tales como: televisión digital interactiva (iDTV), consolas de videojuegos, teléfonos móviles de tercera generación, asistentes digitales personales $(P D A \mathrm{~s})$ y otros. Por tanto, las organizaciones públicas deben llevar a cabo contenidos, servicios y productos susceptibles de ser ofrecidos mediante diferentes plataformas tecnológicas.

\section{Interfaces de acceso avanzados}

Los usuarios con escasa familiaridad con las tecnologías web, o aquellos que presentan algún tipo de discapacidad, deben tener las mismas posibilidades que los más avanzados y, por lo tanto, habrá que implementar interfaces tecnológicos "humanos" que permitan entre otras cosas:

—búsqueda en lenguaje natural y controlado,

-interacción mediante mecanismos de reconocimiento de voz, y

—creación de páginas que puedan ser leídas fácilmente por los lectores automáticos de pantalla.

\section{Personalización de servicios}

Los sitios web deben presentar la información de mayor interés para los usuarios, así como la posibilidad de recibir alertas (mediante e-mail, mensajes a móvil, mensajes iDTV, etc.) para comunicar que se ha publicado nueva información de su interés (servicios push). Esto significa que se deben fomentar desde las organizaciones públicas los procesos de fidelidad y confianza, asegurando que ofrecen información personalizada de interés.

\section{Servicios integrados}

La integración de sitios web que ofrecen servicios de administración electrónica debe ser transparente al usuario. Ha de existir una coordinación entre organizaciones locales, regionales y la Administración central para que se reciban los servicios requeridos sin tener que acudir al sitio web de la organización o departamento que lo suministra.

\section{Servicios transaccionales}

Son el gran reto de la administración electrónica, de forma que el usuario pueda realizar electrónicamente los procesos que requieran la interacción con una organización pública. Muchos de estos servicios ya están disponibles: modificar un registro de datos personales (cambio de dirección), solicitar un documento (solicitud del pasaporte), comprar o pagar algo (para impuestos), etc. El sistema ha de permitir a los ciudadanos realizar un seguimiento del estado en el que se encuentra su tramitación (solicitud, pago, compra, etc.).

\section{Las 10 buenas prácticas en el desarrollo de sitios web}

\section{Que sean atractivos, usables y accesibles}

Los sitios web públicos deben orientarse al usuario, lo cual implica:

-Ofrecer una imagen atractiva que fomente su utilización.

- Proporcionar la información y los servicios que el usuario desea.

—Evolucionar para satisfacer sus necesidades.

- Implementar los conceptos de usabilidad y accesibilidad web.

a. Aspectos importantes. La usabilidad consiste en medir la calidad de la relación del usuario cuando interactúa con el producto o sistema, ya sea un sitio web, una aplicación de software, tecnología móvil, o cualquier otro sistema de interacción. La experiencia de usuario es fundamental para el éxito de un sitio. En ella intervienen diferentes factores, pero lo crucial es la impresión que permanece en ellos, algo muy difícil de analizar.

La accesibilidad web significa que una amplia variedad de software y usuarios sean capaces de acceder y recibir el contenido desarrollado en el sitio web. El $W 3 C$ ha publicado una recomendación internacional con relación a este punto y definida en las Directrices de accesibilidad para el contenido web (Web content accessibility guidelines, Wcag) incluidas en la Iniciativa de accesibilidad web (Web accessibility initiative, WAI).

http://www.w3c.org/WAI 
La comunicación COM (2001) 529 final y la resolución del Consejo (2003/C 39/03) de 6 de febrero sobre accesibilidad electrónica establecen la necesidad de que los sitios web y otras aplicaciones implementen las directrices de accesibilidad.

\section{«Todavía en muchos lugares se entiende como administración electrónica la mejora de la atención al ciudadano median- te el uso de las tecnologías de información y comunicación»}

b. Consejos prácticos. Las investigaciones llevadas a cabo por User Interface Engineering, Inc. muestran que los usuarios no son capaces de encontrar la información que buscan en la web en un $60 \%$ de las ocasiones. Esto puede conducir a una pérdida de tiempo, a la reducción de la productividad, al incremento de la frustración y a la decisión de no volver a visitar el sitio web. Por tanto, en la planificación o creación de nuevos servicios se deben tener en cuenta los principios esenciales de arquitectura de la información y usabilidad web. En este sentido, Jakob Nielsen comenta que "los estudios sobre la conducta de usuarios en la web muestran una baja tolerancia hacia los sitios con dificultades de diseño o extremadamente lentos. Los usuarios no esperan y no quieren aprender cómo usar un sitio web".

\section{Herramientas de validación de W3C}

Este organismo ofrece una serie de directrices técnicas que deben ser aplicadas en el desarrollo de sitios web. Los aspectos básicos se refieren a la normalización tanto del lenguaje de marcado utilizado como de las hojas de estilo y las directrices de accesibilidad web.

-El lenguaje de marcado: se proporciona una aplicación para validar si el código fuente de un documento html cumple las directrices de un lenguaje de marcado específico; si el sitio web las acata se puede certificar mediante el logo correspondiente.

http://validator.w3.org

- Hojas de estilo: también ofrece una herramienta para validar si cumplen ciertas directrices, certificándose mediante su proceso correspondiente, igual que en el caso anterior.

http://jigsaw.w3.org/css-validator

-Accesibilidad web: W3C propone unas directrices para mejorar este punto. Si el sitio web cumple algunas o todas las directrices de accesibilidad web se puede certificar mediante el logo pertinente.

http://www.w3c.org/WAI

\section{http://www.useit.com}

Las directrices de la Iniciativa de accesibilidad web establecen 3 niveles (A, doble A y triple A) dependiendo del grado de conformidad con las prioridades 1,2 y 3 de las directrices. Los sitios web deben cumplir al menos la prioridad 1 (nivel A) de WAI. Para analizar la accesibilidad se puede utilizar una herramienta en castellano denominada Test de accesibilidad web $(T A W)$, que ha sido llevada a cabo por la Secretaría General de Asuntos Sociales, Imserso, Ceapat y Sidar para el análisis e información del grado de accesibilidad de un sitio web.

http://www.tawdis.net

Además de estas directrices, se deben implementar otras opciones que mejoren la accesibilidad global. Consideramos esenciales los atajos de teclado, los índices de tabulación y los links invisibles.

\section{Trabajo en coordinación con otras organiza- ciones}

Sería necesario un trabajo en armonía con otros organismos públicos para ofrecer mejores servicios a los usuarios.

a. Aspectos importantes. Todos los documentos y servicios públicos electrónicos deberían tener un lugar estable (url), de forma que los portales temáticos, los directorios y los motores de búsqueda puedan indizarlos correctamente para crear enlaces permanentes. Los usuarios no quieren saber qué organismo público ofrece un servicio u otro, sino acceder de una forma intuitiva y sencilla a la información y servicios públicos organizados en torno a sus necesidades esenciales, no a las de la organización.

b. Consejos prácticos. Se debe optar por un nombre de dominio fácilmente reconocible por el usuario; tender a la normalización tal y como ocurre en otros países (por ejemplo, en el Reino Unido todos los sitios web de carácter público poseen el subdominio .gov.uk) para que se pueda intuir la dirección url de las organizaciones públicas (Ciberp@is, 2001).

Hay que desterrar la idea de que nuestro contenido es el único importante y hay que establecer enlaces con otras páginas (Nielsen, 2000, pp. 6-7). Los sitios web deben vincularse con otros igualmente relevantes y realizar links profundos a secciones y/o documentos específicos, no sólo a la página principal.

\section{Servicios para los ciudadanos}

Las organizaciones públicas deben trabajar para proporcionar una versión electrónica de todos los servicios posibles. 


\section{Documentos de interés}

- Libro blanco para la mejora de los servicios públicos: una nueva administración al servicio de los ciudadanos. Ministerio de Administraciones Públicas, 4 de febrero de 2000.

http://www.map.es/docu/libro/pdf.htm

-Fundación Retevisión [Fundación Auna desde mayo de 2002]. Racionalización de las políticas de administración electrónica en la Unión Europea. Estudio encargado por la Presidencia Española del Consejo de la UE. Madrid, 25-26 de abril de 2002.

http://www.map.es/csi/pdfIRacionalEsp.pdf

-Fundación Retevisión. Informe sobre la evolución de los servicios públicos europeos dentro de la Unión Europea. Estudio encargado por la Presidencia Española del Consejo de la UE. Madrid, 25-26 de abril de 2002. http://www.map.es/csi/pdfleGovEsp_definitivo.pdf

-Achieving universal access. London: Booz, Allen \& Hamilton, March 7th 2000. Consultado en: 02-0403.

http://lacnet.unicttaskforce.org/Docs/Booz\%20Allen/Ach ieving\%20Universal\%20Access.pdf

-Fp 6 Integrating Programme. Priority 7: citizens and governance in a knowledge based society. Work Programme 2002-2003. Consultado en: 02-04-03.

http://europe.ish-lyon.cnrs.fr/Extranet/Documents/ WP_Pr7.pdf

\section{Documentos europeos relacionados}

- La información del sector público: un recurso clave para Europa. Libro Verde sobre la información del sector público en la sociedad de la información, COM (1998) 585.

- Comunicación de la Comisión al Consejo, el Parlamento Europeo, el Comité Económico y Social y el Comité de las Regiones. La eEurope 2002: creación de un marco comunitario para la explotación de la información del sector público, COM (200I) 606 final.

- Comunicación de la Comisión al Consejo, el Parlamento Europeo, el Comité Económico y Social y el Comité de las Regiones. La eEurope 2002: accesibilidad de los sitios web públicos y de su contenido.

- Comunicación de la Comisión al Consejo, el Parlamento Europeo, el Comité Económico y Social y el Comité de las Regiones. La eEurope 2005: una sociedad de la información para todos, COM (2002) 265 final.

-Resolución del Consejo de 6 de febrero de 2003 sobre accesibilidad electrónica - Mejorar el acceso a las personas con discapacidad a la sociedad del conocimiento (2003/C 39/03).

\section{Normativa aplicable}

- La normativa aplicable en el desarrollo de sitios web debe ser analizada y tenida en cuenta, se debe extraer un conjunto de especificaciones técnicas objeto de implementación.

\section{Normativa sobre utilización de técnicas electrónicas, informáticas y telemáticas por la Administración General del Estado}

a. Legislación Europea.

-Directiva 2000//3/CE, de 8 de junio, relativa a determinados aspectos jurídicos de los servicios de la sociedad de la información, en particular el comercio electrónico y el mercado interior (Directiva sobre el comercio electrónico). Doce 17-07-2000.

b. Legislación nacional.

- Ley 34/2002, de II de julio, de servicios de la sociedad de la información y del comercio electrónico, BOE 12-07-02.

-Real Decreto 263/1996 de 16 de febrero, regula la utilización de técnicas electrónicas, informáticas y telemáticas por la Administración General del Estado, BOE 19-02-1996.

\section{Normativa sobre protección de datos de carácter personal}

a. Legislación Europea.

-Directiva 2002/58/CE, de 12 de julio, relativa al tratamiento de los datos personales y a la protección de la intimidad en el sector de las comunicaciones electrónicas (directiva sobre privacidad y las comunicaciones electrónicas), Doce 31-07-2002.

b. Legislación nacional

- Ley Orgánica 15/1999, de 13 de diciembre, de protección de datos de carácter personal (deroga la Ley Orgánica 5//992, Lortad), BOE I4-12-1999.

\section{Normativa sobre bases de datos}

-Directiva 96/9/CE, de II de marzo de 1996, sobre la protección jurídica de las bases de datos. Doce $L$ 077 de 27-03-1996. 


\section{a. Atajos de teclado}

Ayudan sobre todo a los usuarios que tienen problemas en el uso de dispositivos de apuntamiento como el ratón. Se realizan mediante el atributo accesskey, que ha sido introducido en $\mathrm{html} 4.0$ con el objetivo de proporcionar una forma alternativa de navegación y se debe añadir al elemento link, como en el siguiente ejemplo:

$<a$ ref="pagina.html" accesskey="|">Página de inicio $<|a\rangle$

Se activan de forma diferente dependiendo del tipo de navegador que se utilice:

-Con Internet Explorer 4 se debe presionar "alt" y la letra/número correspondiente de forma simultánea.

- Con Internet Explorer 5 y superior se ha de presionar "alt", la letra/número y después "intro".

-Con Netscape 6 y superior se debe presionar "alt" y la letra/número correspondiente.

Se ha realizado una adaptación de UK Government accesskey standard, publicado en Guidelines for UK Government websites, mayo de 2002, para normalizar los atajos de teclado invisibles. Sería recomendable que existiera un consenso para normalizar esta cuestión en todos los sitios web públicos de nuestro país de forma que los usuarios pudieran utilizar la misma combinación de teclas en todos ellos.

Los atajos de teclado visibles llevan a las 3 áreas de contenidos y se visualizan mediante una letra subrayada en la barra de navegación principal.

- p: profesionales.

-r: recursos.

—d: bases de datos.

Los atajos de teclado invisibles son los siguientes:

- c: ir a la sección de contenidos.

-I: página de inicio.

-2: noticias.

a. Aspectos importantes. Es necesario identificar los servicios más demandados por los usuarios y aquellos que aportan la mejor relación coste-beneficio para la propia organización. El objetivo consiste en ofrecer los que los usuarios quieren usar. La oferta de servicios online debe repercutir de forma directa en los procesos de gestión de toda la organización. Se trata de unir la gestión interna (back-office) con la oferta de servicios vía web (front-office).
-3: mapa del sitio.

4: opciones de búsqueda.

-5: quiénes somos.

-6: ayuda.

-7: nota de responsabilidad.

-8: aviso legal.

-9: contacto.

-0: atajos de teclado.

\section{b. Índices de tabulación}

Permiten establecer un orden en la navegación que se realiza mediante tabuladores $y$, además, se deben crear índices de tabulación para todos los formularios; su práctica debe ser racionalizada. Se realizan mediante el atributo tabindex, que establece el orden en el que el cursor se desplazará cada vez que presionemos la tecla de tabulación y debe utilizarse de la forma que aparece en el siguiente ejemplo:

$<$ form $>$

$<p>$ Nombre: <input tabindex="|" type="text" name="nombre" $><b r>$

$<p>$ E-mail: <input tabindex="2" type="text" name $=$ "correo" $>\langle b r\rangle$

\section{c. Links invisibles para los usuarios ciegos}

Los lectores automáticos de pantalla detectan estos enlaces que ayudan a los usuarios invidentes a mejorar la navegación. Al inicio de cada página debe existir un conjunto de enlaces de orientación. Es esencial uno que apunte a la sección de contenidos para que estos usuarios no tengan que escuchar toda la barra de navegación cada vez que cambian de página. Además habrá un vínculo a la página de accesibilidad web, donde se expliquen las diferentes opciones que se han incorporado en este sentido. Finalmente, es recomendable otro a la página de inicio, para que los usuarios puedan iniciar un nuevo proceso de navegación.

b. Consejos prácticos. Las organizaciones públicas deben intentar implementar en versión electrónica la totalidad de los servicios que ofrecen de forma directa y no limitarse a los 20 servicios públicos básicos propuestos por el Consejo Europeo en marzo de 2001 (Fundación Retevisión, 2002b). Deben utilizar técnicas de $C R M$ (gestión de la relación con el cliente) para obtener información sobre la conducta del usuario y poder redefinir los servicios existentes y/o desarrollar otros nuevos que se adapten a las necesidades reales de 
los ciudadanos (Lara y Martínez, 2002a, pp. 408-420).

Algunas herramientas para saber lo que piensan los usuarios de nuestros servicios y productos electrónicos son los ficheros logs y las cookies. Para la gestión eficiente de estos datos existen ciertos programas de software que facilitan su tratamiento. Uno de ellos es Webtrends Traffic Analysis (figura 1).

\section{Contenido efectivo}

Los materiales de los sitios web de carácter público deben ofrecer garantías de calidad, precisión, uniformidad y coherencia.

a. Aspectos importantes. Los web públicos contienen gran cantidad de documentos que han de estar

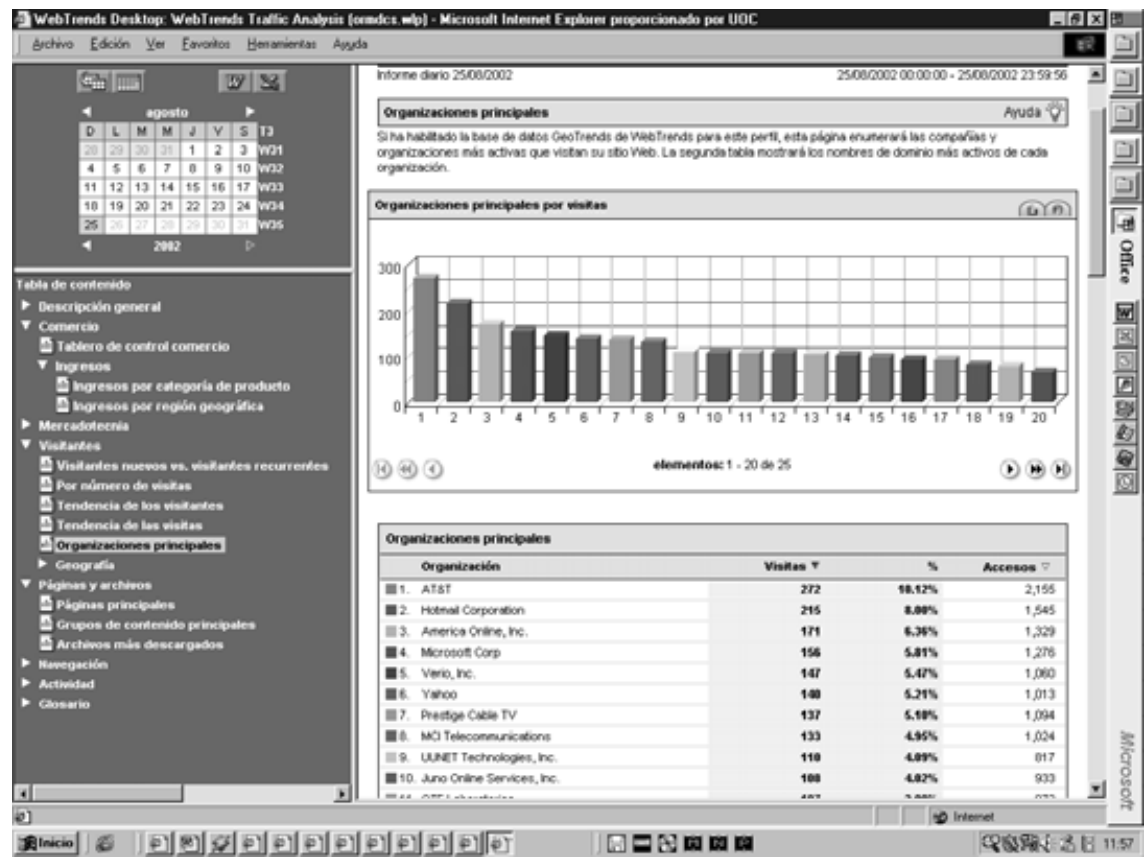

Figura 1 bien organizados, actualizados y mantenidos correctamente. Sus autores y los gestores del sitio web deben generar documentos específicos para cada tipo de usuario, así como crear materiales que faciliten su lectura en línea.

b. Consejos prácticos. Sería necesaria una serie de contenidos mínimos, entre ellos: detalle de la estructura organizativa, los objetivos de la organización, datos completos de contacto, procedimientos de queja y/o sugerencia, noticias y documentos generados por el organismo. La fecha de creación y última actualización debería reflejarse en cada uno de los documentos publicados.

\section{Fomento de la confianza}

Se debe conseguir la confianza de todos los usuarios y para ello habrá que cumplir con toda la normativa aplicable para el suministro de contenidos, servicios y productos electrónicos; además de explicar claramente las políticas y condiciones de uso.

\section{«La administración electrónica en la agenda de la UE tiene un gran peso pero está lejos de presentar un enfoque coordi- nado»}

a. Aspectos importantes. Los responsables de la gestión del sitio web estarán informados sobre sus obligaciones legales en la sistematización, publicación y conservación de la información. La organización tendrá una política de seguridad para asegurar que el servidor que lo alberga (tanto si es interno como si se trata de un servicio de "hosting" de un proveedor de

internet) cumple ciertas condiciones de seguridad. Por otra parte, los usuarios deben tener conocimiento expreso del tratamiento que van a recibir sus datos de carácter personal.

b. Consejos prácticos. Sería conveniente el desarrollo de políticas en diferentes áreas: responsabilidad, protección de datos de carácter personal y de copyright, que además deben ser publicadas en el sitio web. Un ejemplo claro de este tipo de prácticas aparece en Portal Mayores.

Todos los sitios web públicos deben tener una etiqueta de la Plataforma para la selección de contenidos en internet, Pics (Platform for internet content selection) del W3C. Este sistema permite obtener el reconocimiento de que los contenidos son adecuados para ciertos tipos de usuarios y, por tanto, el software de control de contenidos (en el hogar, los colegios, el lugar de trabajo, etc.) podrá o no dar acceso a ellos. Se trata de un sistema similar a la clasificación de películas por edades, pero en este caso los ciudadanos pueden filtrar automáticamente las páginas con cierto tipo de materiales.

http://www.w3c.org/PICS

Para llevar a cabo la clasificación de Pics, obtener un código que lo certifique y que se pueda integrar en los metadata de la cabecera de un documento html, es necesario utilizar un servicio de validación. El más difundido es Icra (Internet Content Rating Association). http://www.icra.org

Un ejemplo de código Pics generado mediante el servicio Icra para Portal Mayores es el siguiente: $<$ meta http-equiv="pics-label" content='(pics-1.1 "http://www.icra.org/ratingsv02.html" comment 


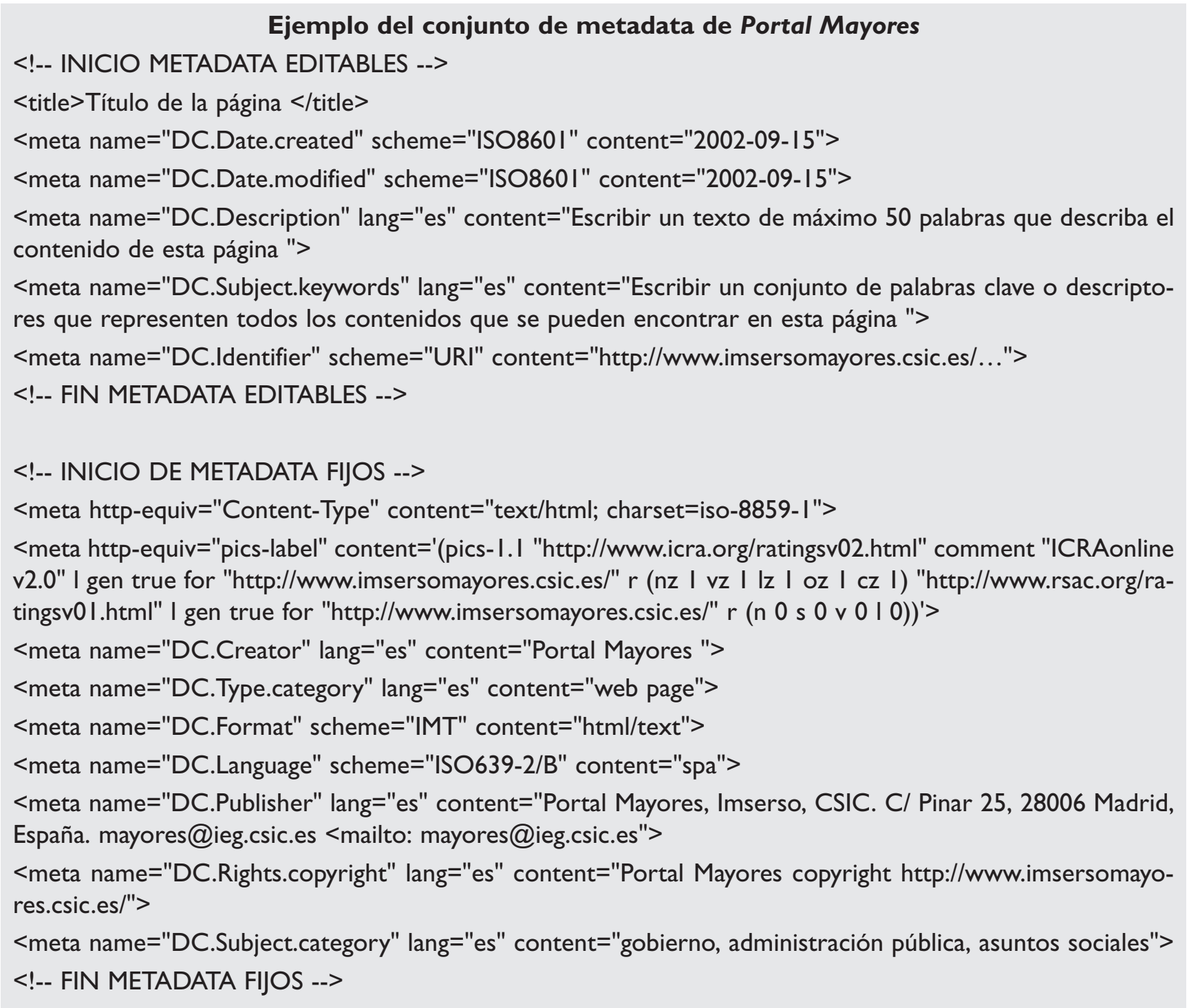

\section{"ICRAonline v2.0" l gen true for}

"http://www.imsersomayores.csic.es/" $r(n z 1 \mathrm{vz} 1 \mathrm{lz} 1$ oz 1 cz 1) "http://www.rsac.org/ratingsv01.html" l gen true for "http://www.imsersomayores.csic.es/" $r$

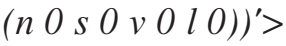

\section{Escuchar a los usuarios}

El usuario espera que la comunicación sea bidireccional y por tanto se proporcionará la oportunidad de contactar, expresar opiniones y realizar consultas a través del web.

a. Aspectos importantes. La organización decidirá la mejor metodología (que recogerá en su política de comunicación) para gestionar las consultas que se reciban de forma electrónica y determinar plazos para la respuesta.

b. Consejos prácticos. La información de contacto estará accesible desde cualquier parte del sitio web. Además, se considera adecuado establecer un correo electrónico genérico que puede aparecer en todas las páginas. Las herramientas para facilitar el contacto de- ben ser lo más sencillas posibles, evitándose los formularios con muchos campos. A veces sólo es necesario incluir la dirección de correo del usuario para solicitar información, suscribirse a ciertos servicios o realizar una consulta.

\section{Proporcionar canales de acceso múltiples}

Hay que facilitar los contenidos mediante diferentes canales tecnológicos, tales como televisión digital y dispositivos móviles.

a. Aspectos importantes. Los sitios web desarrollados con cierta tecnología pueden ser visualizados directamente. Actualmente, ya es posible crearlos para que puedan ser vistos directamente en televisión digital; otros necesitarán ser recodificados de forma automática. Para otros canales, como WAP (wireless application protocol) o dispositivos portátiles, los contenidos y los servicios deben ser creados "a medida".

b. Consejos prácticos. Los sitios de nueva creación deberían utilizar un lenguaje de marcado que permitiera la exportación a diferentes plataformas y que gene- 
rara contenidos "a medida" a partir de cierta codificación. Actualmente, se considera que xml es el ideal para este tipo de aplicaciones.

\section{Evaluación continua}

Deben desarrollarse sistemas que permitan evaluar el éxito obtenido y determinar si se están satisfaciendo las necesidades de los ciudadanos.

a. Aspectos importantes. La organización establecerá procesos que hagan posible recoger información de los usuarios que visitan el sitio web (por ejemplo mediante técnicas y herramientas de $C R M$ y $V R M$ ) y obtener información sobre las necesidades de los que todavía no lo visitan. La eficiencia en los servicios y la mejora continua es un compromiso, puesto que se trata de dinero público, lo cual implica que debe invertirse de forma correcta.

b. Consejos prácticos. Sería conveniente poseer una herramienta de análisis de los ficheros log. Las estadísticas mínimas que se deben extraer son acerca del número de usuarios, la cantidad de visitas, las páginas visualizadas, las peticiones satisfechas y con error, las páginas más y menos visitadas, las que soportan mayor tráfico de entrada y aquellas desde las que llegan nuestros usuarios. Se considera aceptable el uso de cookies para conocer el comportamiento de nuestros usuarios, pero este hecho debe ser claramente reflejado en la política de responsabilidad y el sitio web debe funcionar incluso cuando hayan sido desactivadas.

\section{Promoción del sitio web}

Los sitios web públicos deben contener metadata consistentes que deben usarse para su promoción y registro en los motores de búsqueda.

a. Aspectos importantes. Deben ser objeto de un conjunto de actividades de promoción, tanto las tradicionales (inclusión de la url en los documentos, folletos, publicidad, etc.) como en las electrónicas (intercambio de links con otras organizaciones, compra de publicidad electrónica, registro en portales temáticos, directorios y motores de búsqueda, planes de posicionamiento web, etc.).

La creación de metadata sería conveniente que fuera descentralizada, para que los autores de los documentos pudieran ayudar a proporcionarlos. Ha de tenerse en cuenta que no sólo cada página html debe contener metadata, ya existen métodos (mediante las propiedades de cada sistema) para crearlos en documentos creados con Adobe Acrobat y Microsoft Word.

b. Consejos prácticos. Existen directrices para crear los metadata de los sitios web públicos. Por ejemplo, Dublin Core ha desarrollado una adaptación de- nominada Government Application Profile, disponible en:

http://dublincore.org/groups/government/profile200111.shtml

Para la producción de metadata normalizados se puede pedir consejo a los documentalistas de la organización, expertos en clasificación y representación del conocimiento. Además, se recomienda el uso de tesauros o listas de vocabulario controlado para la generación de ciertos campos.

\section{«Las organizaciones públicas deben crear contenidos, servi- cios y productos susceptibles de ser ofrecidos mediante di- ferentes plataformas tecnoló- gicas»}

El registro del sitio web en motores de búsqueda debe efectuarse tras haber llevado a cabo un proceso de creación u optimización de los metadata y puede realizarse de forma interna (los gestores del sitio web) o bien externalizarlo y encargarlo a una empresa especializada, que generalmente maximiza el resultado que se conseguiría internamente.

\section{Gestión de los productos y servicios}

Los sitios web públicos deben ser gestionados con las siguientes premisas:

- Los recursos (humanos, técnicos y económicos) adecuados.

- Los procedimientos de realización de tareas y de publicación de contenidos consensuados y conocidos por todo el equipo de trabajo.

-Una estrategia organizativa, objetivos claros y usuarios bien identificados.

-Una estrategia de desarrollo futuro en la que generalmente se debe incluir la evolución hacia bases de datos dinámicas, la utilización de un sistema para la gestión de contenidos web y la creación de contenidos para diferentes canales y plataformas tecnológicas.

a. Aspectos importantes. La gestión de un sitio web es compleja y requiere conocimientos muy diversos. Se consideran esenciales las siguientes facultades en la creación de un equipo de gestión: habilidades de comunicación, conocimientos sobre desarrollo de contenidos, sobre tecnologías de la información, sobre arquitectura de la información y de los procesos de negocio.

b. Consejos prácticos. El sitio web debe desarrollarse de una forma coherente teniendo en cuenta los aspectos técnicos, editoriales, de usabilidad y accesibi- 


\section{Complete su colección de IWE/EPI}

Deseo recibir las siguientes publicaciones:

$\square$ ejemplares de la Bibliografía IWE/EPI I992-200 I por correo postal impresos en papel (gratis).

la Bibliografía IWE/EPI 1992-200I por correo electrónico en formato PDF (gratis).

$\square$ álbumes de cd-rom con el texto íntegro de los primeros 100 números de la revista a 30 euros cada disco (uno es gratis para los suscriptores).

$\square$ _ ejemplares de los siguientes números atrasados de la revista (por favor, envíenme presupuesto).

Nombre:

Institución:

Dpto.:

NIF institucional:

Dirección:

Código postal:

Ciudad:

País:

Teléfono:

Fax:

Correo-e:

Envíe este boletín por correo postal, fax o correo electrónico, a esta dirección:

Swets Blackwell

Caspe, 46

08010 Barcelona

España

Tel.: +34-932 70। 144; fax: 93270 I l45

mnzang@es.swetsblackwell.com

lidad web (Lara y Martínez, 2002b). Se desarrollará teniendo en cuenta un conjunto de buenas prácticas, utilizará normativa internacional y validación tecnológica $(W 3 C)$. Todo el equipo que participe de alguna forma en este proceso tendrá conexión permanente a internet y recibirá formación adecuada sobre la preparación de documentos para ser publicados en él. Además se establecerán las herramientas técnicas que permitan a los autores de contenidos la actualización directa de la información que ellos producen.

\section{Bibliografía}

Ciberp@is, 2001, 5 de julio.

http://www.ciberpais.elpais.es/d/20010705/cibersoc/portada.htm

Devadoss, Paul Raj. [et al.]. "Structurational analysis of e-government initiatives: a case study of SCO". En: Decision support systems, 2002, n 34 , pp. 243-269.

Fundación Retevisión. e-España 2002: situación de España ante la sociedad de la información. 2002a.

http://www.fundacionretevision.es/areas/25 publicaciones/publi_251_2.asp

Fundación Retevisión Auna. Informe sobre la evolución de los servicios públicos europeos dentro de la Unión Europea. Estudio encargado por la
Presidencia Española del Consejo de la UE. Madrid, 2002b, 25-26 de abril.

http://www.map.es/csi/pdfleGovEsp_definitivo.pdf

Lara Navarra, Pablo; Martínez Usero, José Ángel. "Comercio electrónico: la fidelización del usuario". En: El profesional de la información, 2002a, noviembre-diciembre, v. 11, n. 6, pp. 408-420.

Lara Navarra, Pablo; Martínez Usero, José Ángel. "Del comercio electrónico a la administración electrónica: tecnologías y metodologías para la gestión de información". En: El profesional de la información, 2002b, noviembre-diciembre, v. 11, n. 6, pp. 421-435.

Lenk, K; Traunmuller, R. Presentation at the Ifip WG 8.5 Working Conference on Advances in Electronic Government, 2000.

Marchionini, Gary; Samet, Hanan; Brandt, Larry. "Digital Government". En: Communications of the ACM, 2003, v. 46, n. 1, pp. 25-27.

Nielsen, Jakob. Designing web usability: the practice of simplicity. Madrid: Alhambra, 2000.

"¿Qué es el e-gobierno?". En: elprincipe.com. http://www.elprincipe.com/egobierno/indexl.shtml

Relyea, Harold, C. "E-gov: introduction and overview". En: Government information quarterly, 2002, n. 19, pp. 9-35.

Pablo Lara Navarra, Universitat Oberta de Catalunya. plara@uoc.edu José Ángel Martínez Usero, Portal Mayores, CSIC. jamartin@ieg.csic.es 